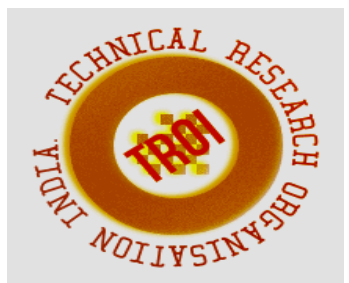

\title{
IOT ENABLED CARBON DIOXIDE AND CARBON MONOXIDE MONITORING AND CONTROL TO REDUCE AIR DETERIORATION FROM VEHICLES
}

\author{
Ashwini. ${ }^{1}$, Balaji. ${ }^{2}$, Naveen. ${ }^{3}$, Sweeta. $S^{4}$, Dr.Palaniswamy $\mathrm{K} \mathrm{M}^{5}$ \\ 1,2,3,4 Student, Guide ${ }^{5}$, Electronics \& Communication Engineering Department, \\ Dr.T. Thimmaiah Institute of Technology, Oorgaum, KGF. \\ Email:ashwinimay1997@gmail.com¹, balajibharadwaj1234@gmail.com², \\ naveenrk1524@gmail.com ${ }^{3}$, sweetathanish@gmail.com ${ }^{4}$,drpalaniswamy@drttit.edu.in ${ }^{5}$
}

\begin{abstract}
Due to the increase in the amount of heat trapping gases, the earth is getting Warm day by day, thus leads to global warming. $\mathrm{CO}$ and $\mathrm{CO}_{2}$ are the main types greenhouse gases. The main goal of this paper is to reduce the greenhouse effect by real time monitoring and controlling of $\mathrm{CO} 2$ and $\mathrm{CO}$ emitted by vehicles and industries using IOT. The Internet of Things (IoT) provides internet connectivity to a various devices and everyday things that use embedded technology to communicate and interact with the outside environment. In this paper, Here the $\mathrm{CO} 2$ and $\mathrm{CO}$ detector intelligent is used to save the $\mathrm{CO} 2$ and $\mathrm{CO}$ levels in different areas. The model is cost effective and can be easily manufactured and installation is possible where ever it is necessary.

Index Terms: Global warming, Greenhouse effect, Internet of Things (IoT), Environment.
\end{abstract}

\section{INTRODUCTION}

Environmental problem arises due to air pollutants from vehicles and trucks are growing rapidly day by day, which are creating respiratory diseases and skin diseases. Transportation alone creates responsible for $50 \%$ of carbon monoxide in the air. This carbon monoxide(CO) shows degradation impact on human health such as chronic obstructive pulmonary disease (COPD) and increases the cause for cancer. The effect of air pollution is very large in cities such that $70 \%$ of the total air pollution has been polluted due to vehicles.
All vehicles emits gases, and the problem occurs when the emission is beyond the standardized values. The main reason for this abnormal emission level is due to incomplete combustion of fuel supplied to the engine

\section{LITERATURE SURVEY}

Prachi Shahane, Preeti Godabole et al [1] have explained that due to rise in the amount of heat trapping gases day by day the earth gets warmer thus leading to global warming. CO2 contributes the maximum pollution in greenhouse gases the main aim of this project is to reduce the green effect by real time monitoring and controlling of $\mathrm{CO} 2$ emission using intellectual IoT. The internet of things (IOT) extends internet connectivity to a multiple range of devices that apply embedded technology to connect and interact with the external environment, all via internet.

P. vlacheas, R. Giaffreda, V. stavroulaki, et al [2] IOT has given an article for upcoming smart cities. In this article the main issues that may prevent IOT from playing this critical role, such as the heterogeneity among connected objects and the undependable nature of associated services were discussed. A cognitive management frame work for IOT is proposed here as solution for real-world objects.

John A.Stankovic, et al[3] have discussed various topics as sensing, actuation, communication and control become even more sophisticated and ubiquitous Here it is shown that how there is a significant overlap in these communities, sometimes from slightly different perspectives.. 
Qihui Wu et al [4] shows how to enable general objects to see, hear, and smell the physical world for themselves as well as through IOT. In this project, it was dicussed that connection alone is not enough, and general objects should have the capacity to link both physical and social worlds by learning, thinking and understanding.

\section{III.RELATED WORK}

In Internet of Things (IoT) era, all day to day events around us are linked to supply chain management through IOT. The application includes healthcare, utilities, transport, etc. The main aim is to make computer sense information without the aid of human intervention is the gist of IoT. IoT converts the information from sensors and connect it to the physical world.

It makes Internet standards to avail services for information transfer, analytics, applications, and communications. Various technologies such as Bluetooth, radio frequency identification (RFID), Wi-Fi, and telephonic data services as well as embedded sensor and actuator nodes. have boosted the development of IoT The cognitive management framework is presented to empower the IoT to better support multiple applications.

Cognition mainly refers to the autonomic selection of the most relevant information for the given application. CIoT is understand as the current IoT integrated with cognitive mechanism to promote intelligence. The eloborate survey shows that from the total CO2 emissions of car $76 \%$ were due to fuel usage, 9\% from manufacturing of the vehicle and $15 \%$ from emissions and losses in the fuel supply system.

\section{IV.PROPOSED WORK}

\section{Arduino Mega 2560:}

Arduino can sense and control more of the physical world than your desktop computer. Arduino is an open-source physical computing platform designed in microcontroller board, and a development environment for writing software for the board.

\section{BLOCK DIAGRAM}

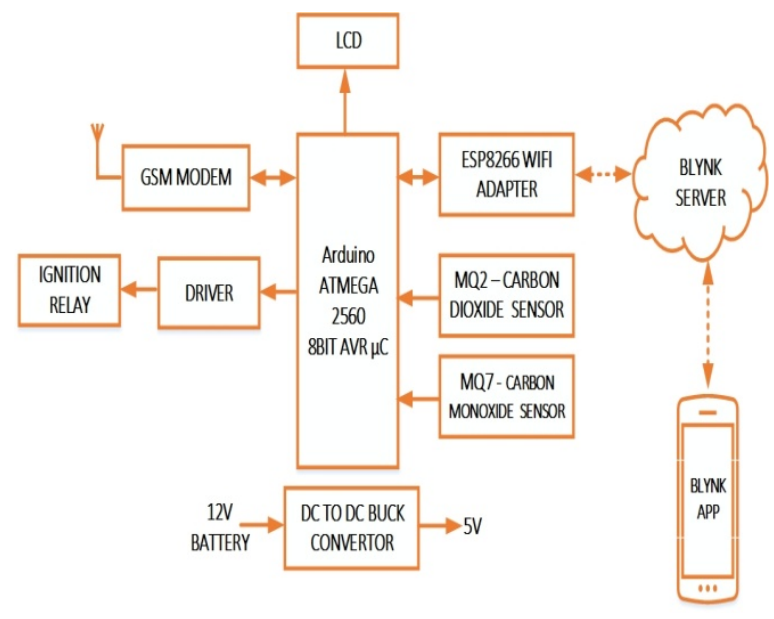

Arduino is designed to take inputs from a variety of switches or sensors, and control variety of lights, motors, and other physical outputs. Arduino projects communicate with software running on your computer (e.g. Flash, Processing, MaxMSP.) The boards can be assembled by hand or purchased preassembled; the open-source IDE can be downloaded for free.

\section{X2 LCD:}

The Liquid Crystal Display (LCD) , low-power and low cost device can displays text and images. LCDs are common in embedded systems where they do not have video monitors like desktop systems. It can be found in numerous common devices like machines and calculators.

\section{GSM:}

GSM900A is a Quad-band type GSM/GPRS Modem which uses frequencies at 850MHz, $900 \mathrm{MHz}, 1800 \mathrm{MHz}$ and $1900 \mathrm{MHz}$. SIM900A can fit almost all application, such as Smart phone, PDA phone and other mobile device. The physical interface to the mobile application is made through a 60 pins board-toboard connector, which provides all hardware interfaces between the module and customers' boards except the RF antenna interface.

The SIM900A is designed with power saving technique of up to as low as $2.5 \mathrm{~mA}$ in SLEEP mode. The SIM800A is integrated with the TCP/IP protocol. Extended TCP/IP AT commands are developed for customers to use the TCP/IP protocol easily, which is very useful for those data transfer applications. 


\section{$\mathrm{CO}_{2}$ Sensor:}

The MQ-135 sensor is more sensitive to CO2 and less sensitive to alcohol and CO. It finds its application in air quality control, ferment process and in-door air monitoring application. The output voltage falls as the concentration of the $\mathrm{CO}_{2}$ increases and viceversa.

\section{CO Sensor:}

MQ-7 gas sensor is high sensitive to Carbon Monoxide. The sensor can be used to detect different mixture of gases gases which contains CO, it is also low cost and suitable for multiple applications.

\section{Ethernet Shield:}

The Arduino Ethernet Shield will connects the Arduino board to IOT. It is based on the WiznetW5100 ethernet chip (datasheet). It uses Ethernet library to write sketches and connect to the internet.

\section{Server Module:}

\section{V.DESIGN FLOW}

Thousands of smart objects with sensors are immersed in the environment, which senses, interfaces, and cooperates with each other to enable efficient services making the economy and the society as a whole. The central cloud/Board receives alerts and respective credentials from millions of vehicles. The amount of data collected at the central board will be very large. The costeffective solution to this is cloud. The data generated at the central board is transmitted to the cloud. The data available at the central server is analysed using Data Analytics Algorithm. The abstracted data will receive the number of alerts of a particular vehicle over a period of time say one month. If the no of alerts about a particular vehicle exceeds the threshold over a period of time, heavy fine can be levied on the owner. The abstracted data also helps the RTO or environmental office for evaluation of the vehicles.

\section{Owner Module:}

All vehicle owner are ensured to install a designed $\mathrm{CO} 2$ and $\mathrm{CO}$ detector at the exhaust to sense the level of $\mathrm{CO} 2$ and $\mathrm{CO}$ emitted in terms of PPM. The $\mathrm{CO} 2$ and $\mathrm{CO}$ sensor will communicates with the Arduino in the vehicle. If the $\mathrm{CO} 2$ and $\mathrm{CO}$ level exceeds the normal PPM level (600 PPM) the Arduino sends a notification to the registered mobile of the owner. This notification is like a warning message given to the owner so that owner is allowed to rectify the vehicle.

If the owner fails to take action to reduce the emissions, an alert signal will be sent to the central board via GSM module on the Arduino. This data acts as useful database to take decisions about the owner at the central board. The decision would be central board will plays a major step in reducing greenhouse effect. If the alerts at the central board exceeds threshold (T1) then the central board would sends the warning message to the owner that the message is likely to turn off the ignition in the vehicle. If The average no of the alerts taken over a period of time exceeds threshold (T2) then automatically it connects to the RTO office to stop the ignition of the vehicle to the BLYNK software.

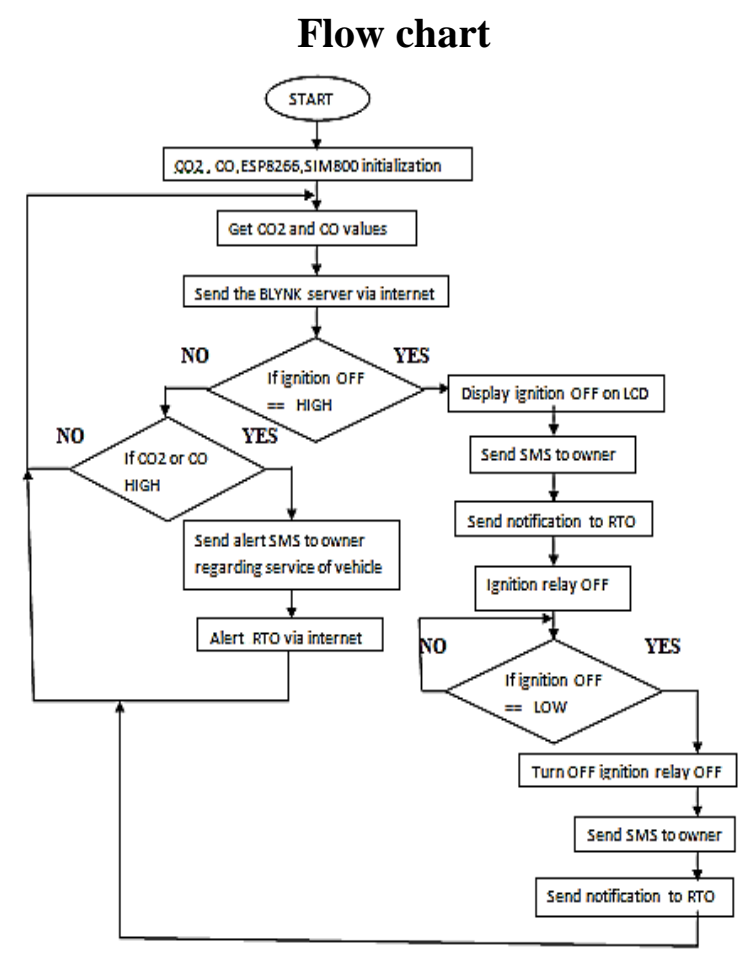

\section{VI.FUTURE SCOPE}

The proposed model only detects carbon dioxide and carbon dioxide. which can also be extended to detect methane, nitrous oxide etc which cause harm to our precious environment. The sensor currently used is MQ135 for detection of CO2 emission and MQ7 for detection of $\mathrm{CO}$ emission. It can withstand a temperature up to 70 degrees. The entire system can be installed in the exhaust of the vehicle. The prototype can be utilized in industries to measure the harmful gases to reduce air pollution caused due to these gases. 
V11. Result

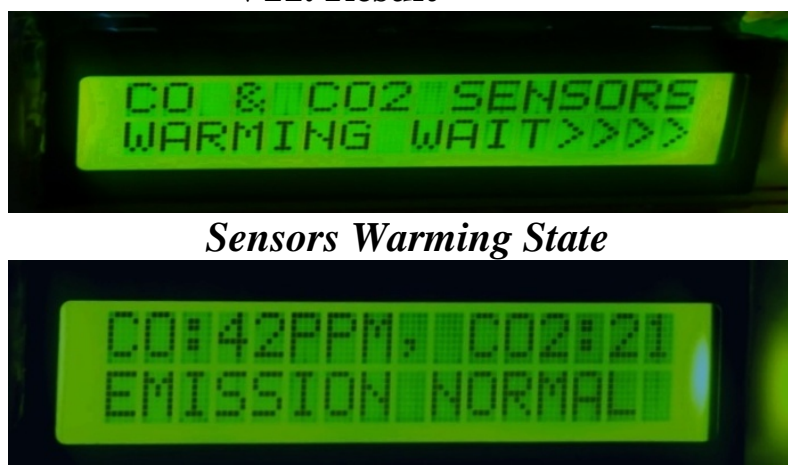

Vehicle Emission is Normal

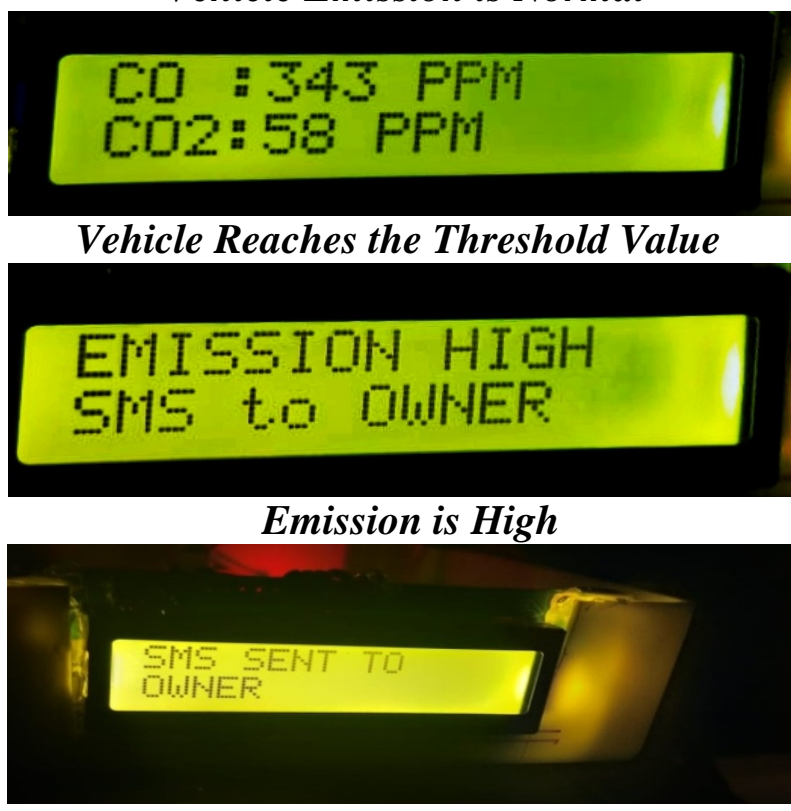

SMS Sent to Vehicle Owner

ALERT UR VEHICLE EMISSION IS EXCEEDING LIMITS,CO:472 PPM, CO2:157 PPM,IGNITION TURNED OFF BY R.T.O.,Service UR Vehicle at the earliest

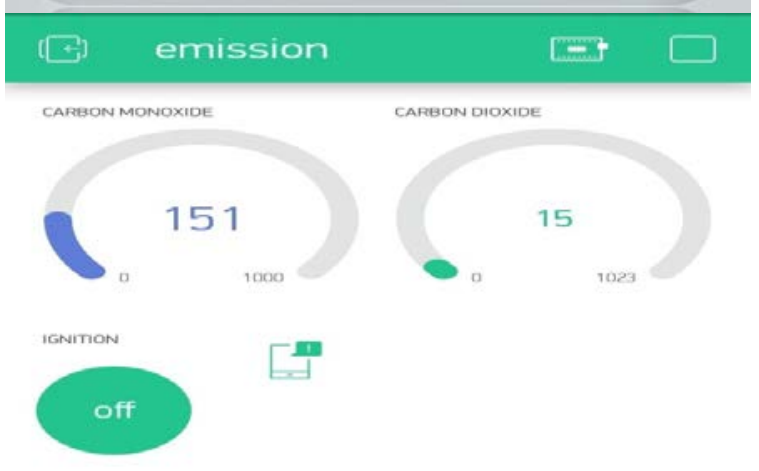

Emission is on that RTO Switch of the Vehicle Ignition

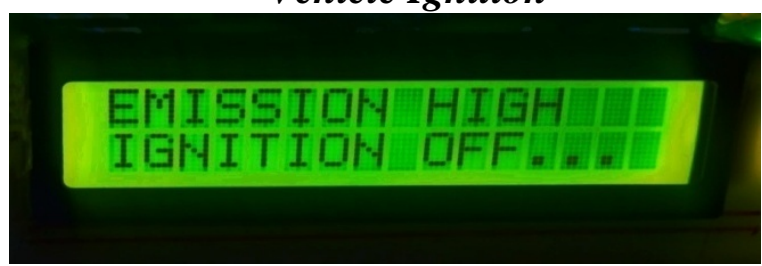

Ignition Off Condition Shown in LCD

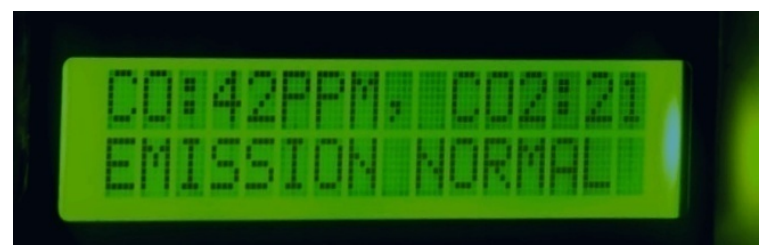

After Seviceing the Vehical the Emission Level Will Normal

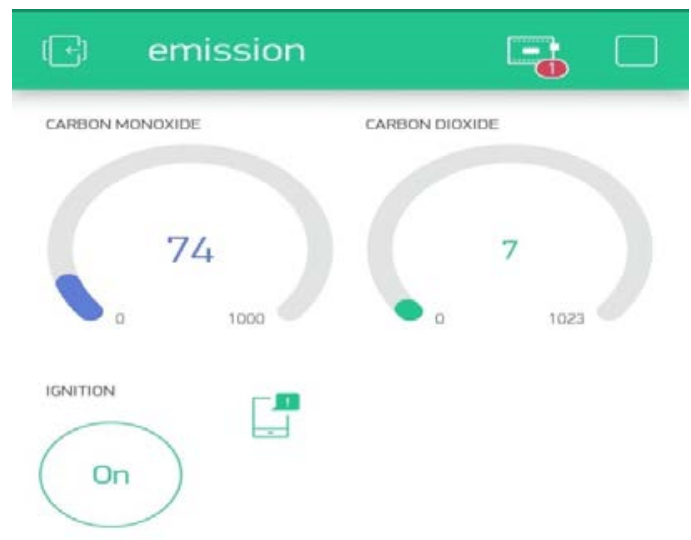

Emission is Normal Vehicle Turn On by RTO

IX. CONCLUSION

Pollution Check has be done atleast once in a month. The model adheres to real time detection of $\mathrm{CO} 2$ and $\mathrm{CO}$ which can reduce the greenhouse gas in the environment. This product can decrease and control the emission considerably thus reduces Global Warming,

\section{REFERENCES}

[1] Prachi Shahane, Preeti Godabole, "Real Time Monitoring of CO2 Emission in vehicle using cognitive IOT", International journal of science and research (IJSR), ISSN (online):23197094, Index Copernicus value (2013):6.14| Impact factor (2014):5.611.

[2] P. vlacheas , R. Giaffreda, V. stavroulaki, et al, "enabling smart cities through a cognitive management frame work for the internet of things" IEEE communications magazine, vol.51,no.6, pp.102-111,june 2013

[3] "Research Directions for the Internet Of Things", John A.Stankovic, Life Fellow, published In March 2014,In Internet Of Things IEEE journalwith ISSN: 23274662 